\title{
Monitoring des Beatmungspatienten
}

\author{
Monitoring of Patients Receiving Mechanical Ventilation
}

Autoren

Institute
J. H. Storre ${ }^{1}$, D. Dellweg

${ }^{1}$ Lungenklinik Köln-Merheim, Kliniken der Stadt Köln gGmbH, Lehrkrankenhaus der Universität Witten/Herdecke, Köln

2 Fachkrankenhaus Kloster Grafschaft GmbH, Lehrkrankenhaus der Philipps-Universität Marburg, Schmallenberg

\section{Bibliografie}

Dol http://dx.doi.org/

10.1055/s-0034-1365742

Online-Publikation: 9.7.2014

Pneumologie 2014; 68: 532-541

(c) Georg Thieme Verlag KC

Stuttgart · New York

ISSN 0934-8387

Korrespondenzadresse PD Dr. med. Jan Hendrik Storre Lungenklinik Köln-Merheim Kliniken der Stadt Köln gGmbH Lehrkrankenhaus der Universität Witten/Herdecke

Ostmerheimer Str. 200

51109 Köln

storrej@kliniken-koeln.de

Serienherausgeber W. Windisch, Köln

\section{Zusammenfassung}

$\nabla$

Die Überwachung eines beatmungspflichtigen Patienten erfolgt in der Regel auf einer Intensivstation und stellt eine Herausforderung für das gesamte medizinische Personal dar. Es ist zunächst entscheidend, die pathophysiologischen Zusammenhänge der zugrunde liegenden Erkrankung zu verstehen, um die Patienten sicher überwachen und therapieren zu können. Neben der Beobachtung wichtiger klinischer Zeichen stehen in der modernen Medizin verschiedene apparative Verfahren für das Monitoring zur Verfügung. Die diagnostischen Möglichkeiten schließen dabei sowohl invasive als auch nichtinvasive Verfahren ein. Darüber hinaus ist es unverzichtbar, Zusammenhänge zwischen dem Sauerstoffangebot, dem Sauerstoffverbrauch sowie der atemmuskulären Belastung während der Beatmung zu verstehen. Der vorliegende Artikel beschreibt diese Hintergründe und stellt die wichtigsten diagnostischen Verfahren vor, welche im Rahmen der Überwachung beatmeter Patienten eingesetzt werden können.

\section{Einleitung}

Zur Gewährleistung des Gasaustausches benötigt der Mensch neben der Lunge eine intakte Funktion seines Atemorgans, der Atempumpe, welches den Gastransport in die Lunge sicherstellt. Funktionsstörungen sowohl der Lunge als auch der Atempumpe können zu einer respiratorischen Insuffizienz führen, die pathophysiologisch hinsichtlich ihrer Ätiologie unterschieden wird: Bei einer hypoxämischen respiratorischen Insuffizienz (Typ I) liegt ein Versagen der Lunge vor, wohingegen bei der hyperkapnischen respiratorischen Insuffizienz (Typ II) die Atempumpe limitiert ist. Eine respiratorische Insuffizienz kann akut oder chronisch auftreten. Neben der Therapie der zugrunde liegenden Erkrankung erfolgt

\section{Abstract \\ $\nabla$}

Patients undergoing mechanical ventilation are usually treated in the intensive care unit. Monitoring of these patients is challenging for all members of the medical staff. Understanding the aetiology of respiratory failure as well as the pathophysiological principles is essential for appropriate monitoring and treatment. Besides observation of clinical signs, different monitoring methods have become available including invasive and non-invasive diagnostic tools. Furthermore, knowledge about oxygen supply and oxygen consumption as well as respiratory muscle capacities and workload is important. The current article presents an overview of these issues and evaluates different diagnostic tools to monitor ventilator-dependent patients.

bei einer respiratorischen Insuffizienz Typ I die Gabe von Sauerstoff, bei einer respiratorischen Insuffizienz Typ II die mechanische Ventilation $[1,2]$.

Sowohl zur Diagnostik als auch während der Therapie einer respiratorischen Insuffizienz ist eine engmaschige Kontrolle der Patienten notwendig. Im Rahmen einer akuten Erkrankungsphase sollte die Überwachung unter stationären Bedingungen auf einer Intensivstation oder in einer spezialisierten Fachabteilung erfolgen $[3,4]$.

Klinische Zeichen einer respiratorischen Insuffizienz sind vielfältig je nach Erkrankungsverlauf und der zugrunde liegenden Erkrankung. Hierzu gehört zunächst die Anamnese nach Luftnot (Dyspnoe) sowie die Messung der Atemfrequenz, welche sowohl erniedrigt (Bradypnoe) als auch er- 
Tab. 1 Messmethoden des $\mathrm{PCO}_{2}$. Modifiziert nach Storre et al. 2011 [9].

\begin{tabular}{|c|c|c|}
\hline & Vorteile (+) & Nachteile (-) \\
\hline $\begin{array}{l}\text { Blutgasanalyse (BGA) } \\
\text { (arteriell oder kapillär) }\end{array}$ & $\begin{array}{l}\text { - Goldstandard des } \mathrm{PCO}_{2} \\
\text { - weitere Informationen: } \mathrm{PaO}_{2}, \mathrm{PH}, \mathrm{HCO}_{3}^{-}, \mathrm{BE} \text { und } \\
\text { Elektrolyte (arteriell) }\end{array}$ & $\begin{array}{l}\text { - invasiv } \\
\text { - schmerzhaft } \\
\text { - Momentaufnahme des Gasaustausches }\end{array}$ \\
\hline Transkutaner $\mathrm{PCO}_{2}$ & $\begin{array}{l}\text { - kontinuierlich } \\
\text { - nichtinvasiv } \\
\text { - stört nicht die Schlafqualität }\end{array}$ & $\begin{array}{l}\text { - technische Abweichung/Drift } \\
\text { - eingeschränkt bei Vasokonstriktion, Hautödemen } \\
\text { - stabile Messwerte nach ca. } 10-15 \text { min } \\
\text { - nicht ausreichend validiert bei der Akut-NPPV }\end{array}$ \\
\hline End-tidal $\mathrm{PCO}_{2}$ & $\begin{array}{l}\text { - kontinuierlich } \\
\text { - nichtinvasiv } \\
\text { - sofort einsetzbar } \\
\text { - stört nicht die Schlafqualität }\end{array}$ & $\begin{array}{l}\text { - ungenau bei inhomogener Ausatemluft: } z \text {. B. bei Leckagen, } \\
\text { Ventilations- und Perfusions-Missverhältnissen } \\
\text { - nicht validiert bei NPPV }\end{array}$ \\
\hline
\end{tabular}

$\mathrm{BE}=$ Base Excess, $\mathrm{BGA}=$ Blutgasanalyse, $\mathrm{HCO}_{3}^{-}=$Standardbikarbonat, $\mathrm{NPPV}=$ nichtinvasive Überdruckbeatmung, $\mathrm{PaO}_{2}=$ arterieller Sauerstoffpartialdruck, PaCO $\mathrm{Carterieller}_{2}$ Kohlenstoffdioxidpartialdruck, $\mathrm{PCO}_{2}=$ Kohlenstoffdioxidpartialdruck.

höht (Tachypnoe) sein kann. Ein typisches klinisches Bild bei Patienten mit Dyspnoe ist dabei der verstärkte Einsatz der Atemhilfsmuskulatur oder auch respiratorische Alternans. Weitere klinische Zeichen einer respiratorischen Insuffizienz können vielfältig sein, hierzu gehören u.a. eine Bradykardie oder Tachykardie, Agitation, Angst, Kopfschmerzen, Somnolenz, Abgeschlagenheit, Leistungsabfall, psychische Veränderungen, Schlafstörungen, Polyglobulie oder auch Ödeme. Neben der wichtigen klinischen Beobachtung der Patienten stehen verschiedene diagnostische Verfahren zur Verfügung [3,4].

Die in der Folge vorgestellten Messparameter wie Sauerstoffsättigung, Sauerstoff- und Kohlendioxidpartialdruck können die Ergebnisse des Gasaustausches, der Ventilation oder auch einer Beatmungstherapie bewerten. Möchte man die Resultate von Atmung oder Beatmung jedoch genau interpretieren und positiv beeinflussen, so sind detaillierte Kenntnisse über das Sauerstoffangebot, den Sauerstoffverbrauch und auch die Atemmuskelfunktion von entscheidender Bedeutung, welche im Anschluss dargestellt werden. Daraufhin erfolgt eine Einführung in die Interpretation von Beatmungskurven bei nicht-invasiver und invasiver Beatmung.

\section{Invasive Methoden zur Bestimmung des Gasaustausches}

$\nabla$

Die arterielle Blutgasanalyse stellt den Goldstandard zur Analyse des Gasaustausches dar ( Tab. 1) [5-9]. Hierbei wird aus einer Arterie eine Blutprobe gewonnen, die unmittelbar in einem Blutgasanalysator untersucht wird. Die patientennahe Diagnostik (engl. „point of care“) ermöglicht eine schnelle Probenverarbeitung und den Erhalt der Messergebnisse innerhalb weniger Minuten. Gerade im Rahmen der akuten medizinischen Versorgung von Patienten in der Notaufnahme oder auf einer Intensivstation hat dieses Verfahren einen sehr hohen Stellenwert. Sind wiederholte Analysen des Gasaustausches notwendig, wie zum Beispiel im Rahmen eines Aufenthaltes auf einer Intensivstation, kann ein arterieller Verweilkatheter angelegt werden, der einen schnellen Zugriff auf erneute Blutproben ermöglicht. Leitwert einer hypoxämischen respiratorischen Insuffizienz (Typ I) ist ein erniedrigter Sauerstoffpartialdruck $\left(\mathrm{PaO}_{2}\right)$ und eine erniedrigte Sauerstoffsättigung $\left(\mathrm{SaO}_{2}\right)$ im Blut. Kompensatorisch kann als Zeichen der Hyperventilation der Kohlenstoffdioxidpartialdruck $\left(\mathrm{PaCO}_{2}\right)$ erniedrigt sein. Die hyperkapnische respiratorische Insuffizienz (Typ II) ist durch einen Anstieg des $\mathrm{PaCO}_{2}$ gekennzeichnet, wobei

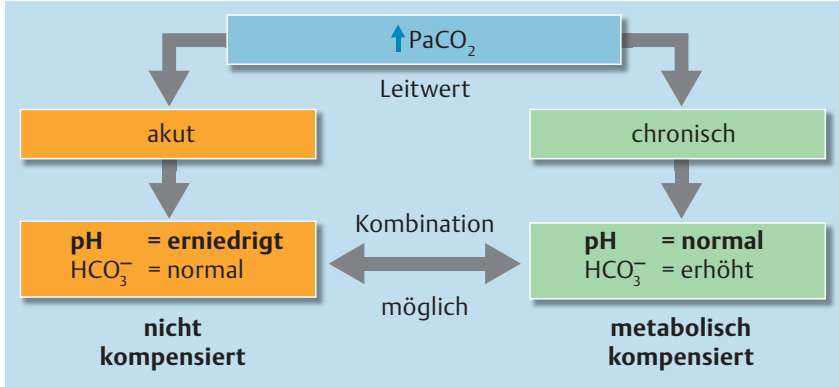

Abb. 1 Hyperkapnische respiratorische Insuffizienz.

auch hier konsekutiv ein erniedrigter $\mathrm{PaO}_{2}$ vorliegen kann [2]. Mit einer arteriellen Blutgasanalyse können diese Parameter genau analysiert werden, zudem kann im Rahmen einer ventilatorischen Insuffizienz durch die gleichzeitige Bestimmung des $\mathrm{pH}-$ Wertes sowie des Bikarbonats und Base-Excess zwischen einer akuten oder chronischen Form der respiratorischen Insuffizienz Typ II unterschieden werden ( $\bullet$ Tab. 1). Ist im Rahmen einer ventilatorischen Insuffizienz der pH-Wert erniedrigt und das Bikarbonat oder Base-Excess im Normbereich, so besteht eine respiratorische Azidose, und es ist von einer akuten Atempumpinsuffizienz auszugehen. Ist der pH-Wert hingegen stabil und liegt ein erhöhtes Bikarbonat oder Base-Excess vor, so ist eine chronische Atempumpinsuffizienz wahrscheinlich ( $\bullet$ Abb. 1). Kombinationen aus akuter und chronischer ventilatorischer Insuffizienz sind ebenso möglich, hierbei zeigt sich ein erniedrigter $\mathrm{pH}$-Wert und ein erhöhtes Bikarbonat [4].

Alternativ zu einer arteriellen Blutprobe kann unter nicht-intensivmedizinischen Bedingungen das arterialisierte Kapillarblut aus dem hyperämisierten Ohrläppchen für die Durchführung einer Blutgasanalyse verwendet werden [7,10,11]. Der Vorteil liegt hierbei in einer geringeren Invasivität, sodass die Abnahmetechnik auch vom geschulten Assistenzpersonal durchgeführt werden kann. Insbesondere für den kapillär gemessenen $\mathrm{pH}$ Wert und den $\mathrm{PaCO}_{2}$ zeigt sich eine sehr gute Übereinstimmung zum Goldstandard aus einer arteriellen Blutgasanalyse. Dagegen ist die Übereinstimmung in den beiden Techniken für den $\mathrm{PaO}_{2}$ weniger genau. Deshalb ist bei hypoxämischer respiratorischer Insuffizienz oder auch unklaren Messergebnissen die arterielle Blutgasanalyse zu favorisieren [7]. Ein weiterer Nachteil liegt in der Zeitspanne von mindestens 10 Minuten, die benötigt wird, um das Ohrläppchen ausreichend zu hyperämisieren [10]. 


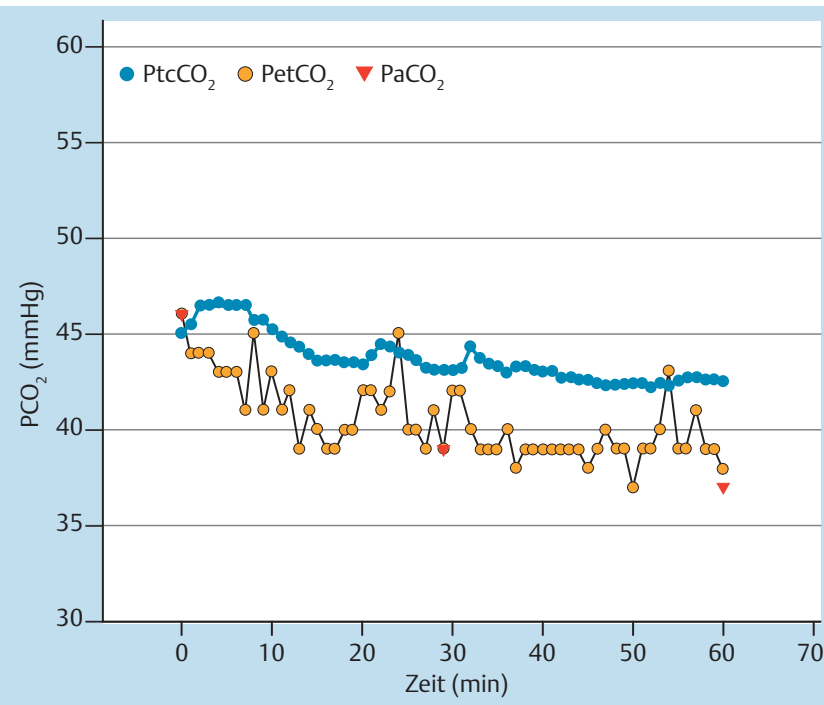

Abb.2 End-tidaler und transkutaner $\mathrm{PCO}_{2}$-Verlauf unter invasiver Beatmung über ein Tracheostoma während des Weaning. 47-jähriger Patient mit thorakal-restriktiver Erkrankung. Beatmungseinstellung: Assistiert/ Kontrollierter Modus, Inspirationsdruck $16 \mathrm{mbar}$, Exspirationsdruck 5 mbar, Atemfrequenz 18/min, Inspirationszeit 1,1 sec, supplementäre Sauerstoffzufuhr 2 Liter $\mathrm{O}_{2} / \mathrm{min}$.

$\mathrm{PaCO}_{2}=$ arterieller Kohlenstoffdioxidpartialdruck, $\mathrm{PetCO}_{2}=$ end-tidaler Kohlenstoffdioxidpartialdruck, $\mathrm{PtcCO}_{2}=$ transkutaner Kohlenstoffdioxidpartialdruck.

\section{Nichtinvasive und kontinuierliche Methoden zur Bestimmung des Gasaustausches}

Die Nachteile einer Blutgasanalyse sind, dass es sich um eine invasive Technik handelt und sie nur eine Momentaufnahme des Gasaustausches widerspiegelt ( $\bullet$ Tab. 1). Aus diesem Grund ist eine verlässliche, nichtinvasive und kontinuierliche Technik wünschenswert $[12,13]$. Eine invasive kontinuierliche Messung des $\mathrm{PaCO}_{2}$ ist zwar seit den 70er Jahren möglich, hat sich jedoch bisher aufgrund von technischen Gründen, hohen Kosten und der Invasivität im klinischen Alltag nicht durchgesetzt, sodass auf den Intensivstationen wiederholte Blutabnahmen zur Durchführung von Blutgasanalysen notwendig sind, um den Verlauf der Ventilation abzubilden [13].

Als nichtinvasive und kontinuierliche Methoden für das Monitoring von Patienten mit respiratorischer Insuffizienz stehen die Pulsoxymetrie sowie die Messung des transkutanen $\left(\mathrm{PtcCO}_{2}\right)$ und end-tidalen $\left(\mathrm{PetCO}_{2}\right)$ Kohlenstoffdioxidpartialdrucks zur Verfügung. Die Einführung der Pulsoxymetrie in den 80er Jahren stellt einen Meilenstein im Monitoring des Gasaustausches dar und hat den Bedarf der arteriellen Blutgasanalysen deutlich reduziert [12]. Bei der Pulsoxymetrie wird die Sauerstoffsättigung nichtinvasiv photometrisch an einem Finger oder Ohrläppchen gemessen. Pulsoxymeter sind heutzutage extrem klein und in Form von batteriebetriebenen Modellen handlich und mobil einsetzbar. Im Bereich der intensivmedizinischen Überwachung, perioperativ oder auch im Rahmen von Interventionen wie Endoskopien ist die Pulsoxymetrie zur Überwachung der Sauerstoffsättigung nicht mehr wegzudenken. Oxygenierungsstörungen können hierbei sehr kurzfristig eintreten und der Sauerstoffbedarf kann bei Patienten schnell schwanken. Gerade in diesen Situationen ist die Pulsoxymetrie ein geeignetes Messverfahren [12]. Allerdings ist sie kein verlässliches und ausreichendes Messinstrument, um Patienten mit ventilatorischer Insuffizienz zu

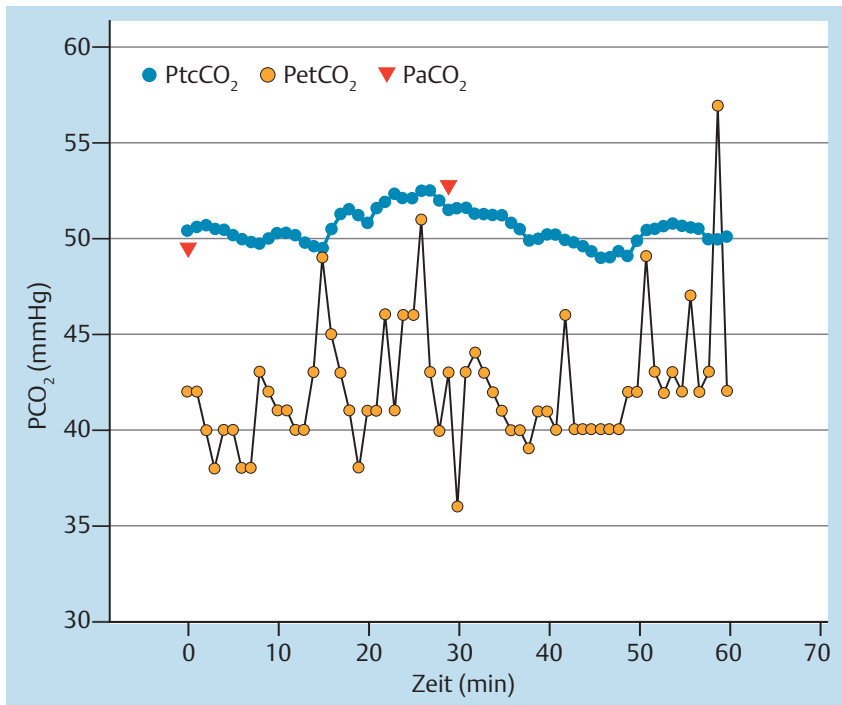

Abb.3 End-tidaler und transkutaner $\mathrm{PCO}_{2}$-Verlauf unter invasiver Beatmung über ein Tracheostoma während des Weaning. 57-jähriger Patient mit COPD. Beatmungseinstellung: Assistiert/Kontrollierter Modus, Inspirationsdruck 21 mbar, Exspirationsdruck 5 mbar, Atemfrequenz 19/min, Inspirationszeit 1,0 sec, supplementäre Sauerstoffzufuhr 2 Liter $\mathrm{O}_{2} / \mathrm{min}$. $\mathrm{PaCO}_{2}=$ arterieller Kohlenstoffdioxidpartialdruck, PetCO ${ }_{2}=$ end-tidaler Kohlenstoff-dioxidpartialdruck, $\mathrm{PtcCO}_{2}=$ transkutaner Kohlenstoffdioxidpartialdruck.

überwachen, denn Normbereiche in der Sauerstoffsättigung schließen eine Insuffizienz der Atempumpe nicht aus. Hierfür muss der Kohlenstoffdioxidpartialdruck gemessen werden [4, 14].

Um diese Patienten kontinuierlich und nichtinvasiv zu monitoren, stehen die Analyse des $\mathrm{PtcCO}_{2}$ und $\mathrm{PetCO}_{2}$ zur Verfügung ( Tab.1, Abb.2-4). Beide Verfahren sind seit Jahren in der klinischen Praxis etabliert. Die Bestimmung des $\mathrm{PtcCO}_{2}$ erfolgt analog der Blutgasanalyse elektro-chemisch in einem Sensor, der auf der Haut über dem Kapillarbett befestigt wird. Ähnlich wie bei der kapillären Blutgasanalyse wird eine Zeitspanne von 10-15 Minuten benötigt, bis das Kapillarblut durch Erwärmung arterialisiert wird ( $\bullet$ Tab. 1). Der wesentliche technische Vorteil des $\mathrm{PtcCO}_{2}$ im Vergleich zur Bestimmung de PetCO $\mathrm{O}_{2}$ besteht darin, dass es bei nicht-beatmeten Patienten und unabhängig vom Beatmungszugang (invasiv/nichtinvasiv) eingesetzt werden kann. Neuere Geräte haben zudem eine Pulsoxymetrie integriert. Das $\mathrm{PtcCO}_{2}$ wurde in mehreren Studien sowohl im Rahmen einer akuten wie auch chronischen ventilatorischen Insuffizienz sowie im Rahmen einer nichtinvasiven Beatmung untersucht $[6,8,13$, 15-17] und wird auch in den entsprechenden Leitlinien der Deutschen Gesellschaft für Pneumologie und Beatmungsmedizin als Messverfahren empfohlen [3,4]. Das $\mathrm{PtcCO}_{2}$ ist in der Lage, die Ventilation im Verlauf sehr gut darzustellen ( $\mathbf{A b b}$.2-4). Dennoch muss gerade im Bereich der Akutmedizin beachtet werden, dass die Messwerte im Vergleich zum $\mathrm{PaCO}_{2}$ in Einzelfällen erheblich schwanken können. Somit kann das $\mathrm{PtcCO}_{2}$ die Blutgasanalyse nicht ersetzen, kann jedoch als ergänzende Messmethode den Bedarf an wiederholten Blutgasanalysen zur Bestimmung der Ventilation reduzieren. Im Bereich der chronischen ventilatorischen Insuffizienz ist das Verfahren validiert und bietet einige wesentliche Vorteile ( Tab.1). Durch das kontinuierliche Monitoring ist beispielsweise eine nächtliche Beatmung deutlich besser abbildbar als durch die Analyse punktueller Blutgasanalysen 


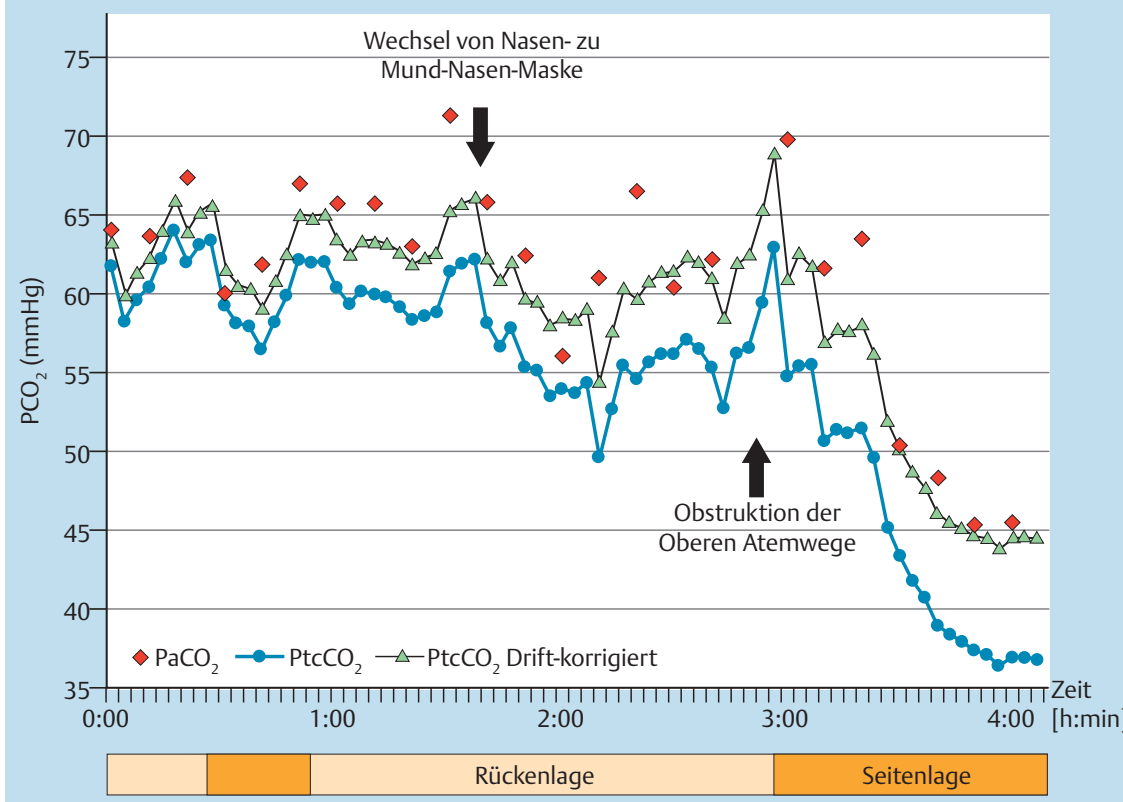

Abb.4 Beatmungseinleitung bei einem 60-jährigen Patienten mit COPD und Adipositas (Body Mass Index $35,4 \mathrm{~kg} / \mathrm{m}^{2}$ ) bei akuter ventilatorischer Insuffizienz. Verlauf des $\mathrm{PaCO}_{2}$ und der Drift-unkorrigierten und Drift-korrigierten $\mathrm{PtcCO}_{2}$-Werte.

Modifizierte Abb. nach Storre et al. [6]. Abdruck mit freundlicher Genehmigung des American College of Chest Physicians.

$\mathrm{PaCO}_{2}=$ arterieller Kohlenstoffdioxidpartialdruck, $\mathrm{PCO}_{2}=$ Kohlenstoffdioxidpartialdruck, $\mathrm{PtcCO}=$ transkutaner Kohlenstoffdioxidpartialdruck.

[8,14]. Zudem müssen Patienten nicht geweckt werden, um eine Blutgasanalyse durchzuführen, was im Rahmen schlafmedizinischer Untersuchungen einen entscheidenden Vorteil darstellt [8]. Die Messung des PetCO ${ }_{2}$ ist vor allem im Rahmen eines operativen Eingriffs im Fachbereich der Anästhesiologie ein verbreitetes Verfahren, um während der Eingriffe und begleitender Narkose die Ventilation zu steuern $[13,15,18]$. Die Bestimmung des Pet$\mathrm{CO}_{2}$ erfolgt am Ende der Exspiration durch eine Infrarotspektroskopie entweder im Haupt- oder Nebenstrom. Häufig ist diese Technik direkt in den Beatmungsgeräten integriert. Die Anwendung erfolgt in der Regel nur bei invasiv beatmeten Patienten. Bei spontan atmenden Patienten hingegen oder unter einer nichtinvasiven Beatmung wird die Technik kaum eingesetzt, da sie unter diesen Bedingungen den $\mathrm{PaCO}_{2}$ nur ungenau abbilden kann $[4,19,20]$. Im Rahmen der nichtinvasiven Beatmung sind häufig Leckagen am Beatmungszugang sowohl während der Inspiration als auch Exspiration zu beobachten [9], weshalb die Technik hier eine Limitation zeigt ( Tab. 1). Zudem ist das Verfahren bei Patienten mit einer Lungenerkrankung, bei denen Ventilations- und Perfusions-Missverhältnisse vorliegen können, bisher kaum validiert und die Messergebnisse können stark schwanken, wie im Beispiel eines COPD-Patienten in $\mathbf{A b b} . \mathbf{z u}$ erkennen ist $[13,15,19]$. Dagegen könnte das Monitoring des $\mathrm{PetCO}_{2}$ bei Lungengesunden ein geeignetes Verfahren sein, den Verlauf einer Ventilation zu beurteilen ( $\bullet$ Abb. 2). Valide Studien liegen hierfür jedoch bisher nicht vor. Zwei Studien, welche das PetCO $\mathrm{O}_{2}$ und $\mathrm{PtcCO}_{2}$ mit dem Goldstandard $\mathrm{PaCO}_{2}$ bei einem Intensivtransport zwischen zwei Krankenhäusern [21] oder bei Kindern mit Herzinsuffizienz [22] miteinander verglichen haben, zeigten, dass das $\mathrm{PetCO}_{2}$ weniger genau den $\mathrm{PaCO}_{2}$ widergespiegelt hat.

\section{Interpretation des Sauerstoffverbrauches}

$\nabla$

Wird eine Beatmung auf Grund einer hypoxämischen Insuffizienz durchgeführt, so sind im Wesentlichen zwei Ziele zu verfolgen:

1. Verbesserung des Sauerstoffangebotes unter Berücksichtigung der Sauerstofftoxizität

2. Reduktion des Sauerstoffverbrauches.

Inspiratorische Sauerstoffkonzentrationen unterhalb von 50\% sind in der Regel nicht toxisch für den Organismus, darüber hinaus kann in Abhängigkeit von Konzentration und Applikationsdauer ein pulmonaler [23] oder extrapulmonaler Schaden [24] induziert werden. Der letale Effekt reinen Sauerstoffs wurde dabei erstmals 1899 tierexperimentell beschrieben [25]. Die Sauerstoffsättigung oder der Sauerstoffpartialdruck alleine sagt nichts darüber aus, ob das Sauerstoffangebot an der Zelle ausreichend ist, da die beiden anderen Organsysteme (Blut und Herz), die am Sauerstofftransport zur Zelle beteiligt sind, hierbei nicht berücksichtigt werden. Um diesen Fehler zu vermeiden, sollte man die Sauerstoffmenge, die pro Zeiteinheit über den Blutweg transportiert wird, berechnen:

$\mathrm{DO}_{2}=\left(\mathrm{Hb} \times 1,34 \times \mathrm{SaO}_{2}+0,003 \times \mathrm{PaO}_{2}\right) \times \mathrm{HZV}[26]$

$\mathrm{DO}_{2}=$ arterielles Sauerstoffangebot, $\mathrm{Hb}=$ Hämoglobingehalt des Blutes, $\mathrm{SaO}_{2}=$ arterielle Sauerstoffsättigung, $\mathrm{PaO}_{2}=$ arterieller Sauerstoff; HZV= Herzzeitvolumen

Selbst wenn auf die Messung des Herzzeitvolumens (HZV) mittels Pulmonaliskatheters oder anderer Verfahren verzichtet wird, ist die Messung des Sauerstoffgehaltes $\mathrm{CaO}_{2}=(\mathrm{Hb} \times 1,34 \times$ $\mathrm{SaO}_{2}+0,003 \times \mathrm{PaO}_{2}$ ) immer noch genauer als die alleinige Bewertung der Oxygenierung des Blutes. Die Normwerte liegen für Männer bei etwa $20 \mathrm{ml} / \mathrm{dl}$, für Frauen bei etwa $19 \mathrm{ml} / \mathrm{dl}$. Werte bis zu $10 \mathrm{ml} / \mathrm{dl}$ können auf zellulärer Ebene problemlos toleriert werden, ohne dass eine Sauerstoffschuld entsteht [27,28]. Sauerstoff liegt im menschlichen Organismus primär an Hämoglobin gebunden vor, ein geringer Anteil ist jedoch im Blut gelöst, wobei letzteres unter klinischen Gesichtspunkten vernachlässigbar ist. Der Sauerstoffverbrauch $\left(\mathrm{VO}_{2}\right)$ und auch die $\mathrm{CO}_{2}$ Abgabe $\left(\mathrm{VCO}_{2}\right)$ des Gesamtorganismus lassen sich über ein metabolisches $\mathrm{Zu}$ satzgerät ähnlich der Spiroergometrie messen [29] oder über 

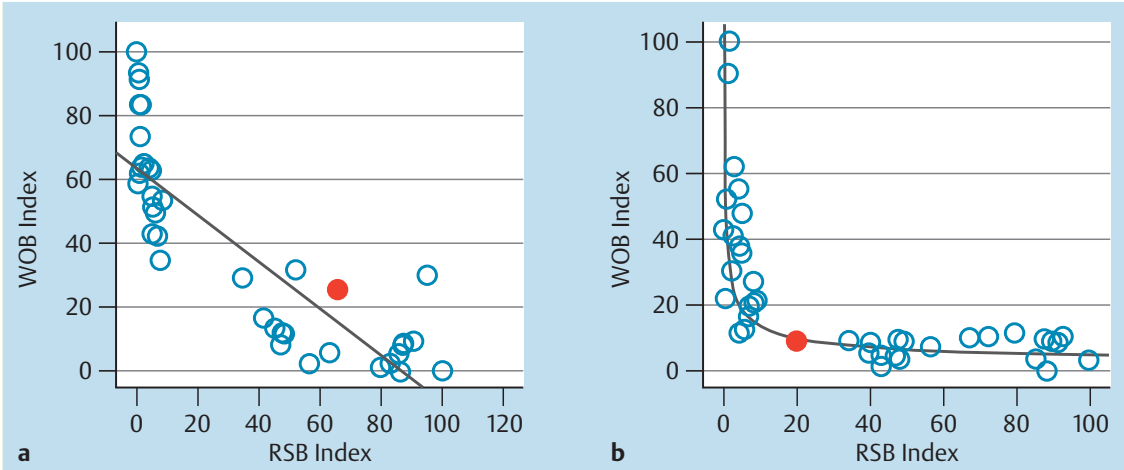

Abb.5 Die Ordinate zeigt die Atemarbeit, der maximale Wert einer Messreihe wurde mit 100 (\%) dargestellt. Der Rapid Shallow Breathing Index (RSB Index) ist der Quotient aus Atemfrequenz und Tidalvolumen. Die Darstellung erfolgt ebenfalls als Index relativ zur Ruheatmung, die durch den schwarzen Punkt gekennzeichnet ist. a gibt die Messwerte Lungengesunder wieder. Der Zusammenhang der Messwerte ist hier linear, während bei Patienten mit COPD (b) eine Zunahme des RSB Index oberhalb der vom Patienten gewählten Atemfrequenz (schwarzer Punkt) keine wesentliche Einsparung der Atemarbeit bewirkt [35].

das Ficksche Prinzip bestimmen (Herzminutenvolumen $\times$ arteriovenöser Differenz des Sauerstoffgehaltes). Teilweise ist die erstgenannte Technik bereits integrierter Bestandteil von Beatmungsgeräten [30]. Das Verfahren misst erst einmal den gesamten Sauerstoffverbrauch des Organismus. Über differentiale Messungen unter Spontanatmung sowie unter maschineller Beatmung lässt sich aber der Sauerstoffverbrauch der Atemmuskulatur (engl. Oxygen Cost of Breathing= OCOB) ermitteln.

Durch die Messung des Sauerstoffverbrauches kann man den Einfluss jeder Variablen bestimmen, so lange man immer nur einen Parameter verändert und dann den Einfluss auf den Sauerstoffverbrauch misst. Auf diese Weise lassen sich die Güte einzelner Beatmungsparameter im Hinblick auf den Sauerstoffverbrauch bestimmen. Auch über die Beatmung hinaus können anderweitige therapeutische Maßnahmen, wie z.B. die Gabe von Katecholaminen, bewertet werden, von denen bekannt ist, dass sie den Sauerstoffverbrauch erhöhen können [31]. Den Sauerstoffverbrauch der Atemmuskulatur durch eine kontrollierte Beatmung zu reduzieren, kann in der akuten Phase einer beatmungspflichtigen Erkrankung von wesentlicher Bedeutung sein. Durch die Arbeiten von Field und Kollegen ist bekannt, dass die OCOB eng mit der Atemarbeit korreliert [32].

\section{Atemmuskuläre Belastung \\ $\nabla$}

Während relative Veränderungen der Atemarbeit durch nichtinvasive Parameter verfolgt werden können, kann eine absolute Messung der Atemarbeit nur über einen Ösophagus- bzw. kombinierten Ösophagus-Magen-Katheter bestimmt werden [33]. Die Parameter der atemmuskulären Belastung können nichtinvasiv und invasiv beurteilt werden.

\section{Nichtinvasive Parameter der atemmuskulären Belastung}

Einfach und ohne großen Messaufwand können Änderungen im Atemmuster wesentliche Informationen über die Belastung geben. Bei belasteter Atemmuskulatur versucht der Organismus die Atemarbeit durch Steigerung der Atemfrequenz bei gleichzeitiger Reduktion des Tidalvolumens (Rapid Shallow Breathing) zu verringern. Yang et al. konnten eindrucksvoll zeigen, dass der Quotient aus Atemfrequenz und Tidalvolumen (Rapid Shallow Breathing Index =RSBI) unter Spontanatmung ein Extubationsversagen vorhersagen konnte, wenn der Index 105 oder mehr betrug [34]. Auf Grund der abnehmenden Compliance und fallender Resistance mit zunehmender Atemzugtiefe ist dieser „Spareffekt“ einer schnellen flachen Atmung abhängig von der Lungenpathologie. Während bei Lungengesunden dieser Zusammenhang line- ar zu sein scheint, verringert sich dieser Benefit bei Patienten mit COPD mit Zunahme der schnellen flachen Atmung ( $\bullet$ Abb.5) [35].

Trotzdem kann eine Änderung des Atemmusters des Patienten hin zum Rapid Shallow Breathing bei assistierten Beatmungsverfahren als atemmuskuläre Entlastungsstrategie des Körpers gewertet werden und auf eine belastete oder überlastete Atemmuskulatur hinweisen. Passend dazu konnte gezeigt werden, dass eine Zunahme der maschinellen Beatmungsunterstützung (Steigerung des Tidalvolumens bzw. des Inspirationsdruckes) zu einer Abnahme der Atemfrequenz [36,37] und des Atemantriebes [38] führt. Bei assistierter Beatmung kann so das Atemmuster Auskunft darüber geben, ob eine veränderte Beatmungseinstellung die Atemmuskeln mehr oder weniger entlastet.

Bei kontrollierter Beatmung hat der Patient dagegen keine Möglichkeit, das Atemmuster (zumindest im Hinblick auf die Atemfrequenz) zu beeinflussen, was das Erkennen der atemmuskulären Be- oder Entlastung erschwert. Eine Asynchronie zwischen Gerät und Maschine (Ventilator Fighting) weist jedoch darauf hin, dass die maschinelle Unterstützung den Bedarf des Patienten nicht abdeckt. Genaueres hierzu finden Sie weiter hinten im Abschnitt über die Interpretation der Beatmungskurven [39].

\section{Invasive Parameter der atemmuskulären Belastung}

Mit Hilfe eines Ösophaguskatheters kann entweder die Atemarbeit (Work of breathing $=$ WOB) oder das Pressure Time Product (PTP) bestimmt werden. Bei der WOB-Messung wird der Ösophagusdruck gegen das Tidalvolumen aufgezeichnet, die Fläche entspricht der Atemarbeit, Werte um 0,7 Joule pro Liter sind beim Lungengesunden normal $[33,35]$. Das PTP errechnet sich aus der Aufzeichnung des Ösophagusdruckes gegen die Zeit und berücksichtigt deshalb auch die atemmuskuläre Arbeit ohne Inspirationsfluss (z.B. Anhalten der Luft am Ende der Inspiration). Aus diesem Grund korreliert das PTP auch am besten mit der OCOB [32]. Ein ansteigendes PTP von mehr als $200 \mathrm{~cm} \mathrm{H}_{2} \mathrm{O}^{*} \mathrm{~s} /$ min konnte in einer Studie von Jubran et al. die Gruppe der Weaningversager identifizieren [40].

Durch tierexperimentelle Daten [41] konnte gezeigt werden, dass eine Unterdrückung der atemmuskulären Aktivität durch kontrollierte Beatmung zu einer atemmuskulären Schwäche führt. Dieses als Ventilator induced diaphragmatic Damage (VIDD) bezeichnete Phänomen konnte in der Folge auch beim Menschen nachgewiesen werden [42]. Auf der anderen Seite führt eine Überlastung der Atemmuskulatur zu strukturellen Schäden [43]. Bei der kritischen Durchsicht der Literatur, die Atemarbeit in Abhängigkeit von der Beatmungseinstellung gemessen hat, bleibt festzustellen, dass bei fehlender Muskelrelaxierung und ohne tiefe Sedierung sich in keiner Studie die Atemarbeit vollkommen 


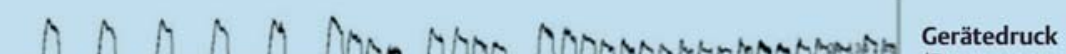 (Daten vom Polygrafiesystem) \\ (n) Bustgurt \\ Gerätedruck \\ (Daten vom Beatmungsgerät)}

Abb. 6 Autotriggern: Der mittlere Kanal zeigt die Atemanstrengung (Brustexkursion) des Patienten. Anhand der beiden anderen Kanäle ist erkennbar, dass es zu einer nicht synchronen und deutlich zu häufigen Druckabgabe durch das Beatmungsgerät kommt. durch die Beatmung unterdrücken ließ [44-48]. Bei der Entstehung des VIDD, bei dem sowohl metabolische Ursachen als auch eine Inaktivitäts-Atrophie diskutiert werden, wird es sich aller Wahrscheinlichkeit nach nicht um ein on/off-Phänomen handeln. Vielmehr wird der Grad der muskulären Inaktivität bzw. Hypoaktivität sowie der Faktor Zeit eine Rolle spielen. Mathematische Modelle hierzu gibt es bisher nicht.

\section{Transpulmonaler Druck (PTP)}

$\nabla$

Seit dem Report des ARDS Network-Trial über den Vorteil der Low-Tidal-Volume Ventilation [49] hat es in Bezug auf die Beatmung beim ARDS wenig positive Ansätze gegeben, die die Beatmungsgüte oder das Outcome wesentlich beeinflussen. Insbesondere Strategien zur Identifikation des besten PEEP-Wertes blieben ohne positives Ergebnis [50-52].

Im Jahr 2008 konnten Talmore und Kollegen dann zeigen, dass eine Titration des PEEP anhand des Transpulmonalen Druckes zu einer besseren Oxygenierung und einem beinahe signifikanten $(p=0,055)$ Überlebensvorteil führte [53]. Die Bestimmung des transpulmonalen Druckes erfolgt ebenfalls mittels Ösophaguskatheter und wird errechnet durch die Differenz zwischen Atemwegsdruck und Ösophagusdruck:

Transpulmonaler Druck (PTP) $=$ (Atemwegsdruck-Ösophagusdruck).

Ziel ist es hier, den Druck in den Atemwegen und den Alveolen gleich dem oder oberhalb des Ösophagusdruckes respektive Pleuradruckes zu halten, um den Alveolarkollaps zu verhindern. In der Studie sollte der transpulmonale Druck in der Inspiration unterhalb von $25 \mathrm{~cm} \mathrm{H}_{2} \mathrm{O}$ liegen und am Ende der Exspiration 0 $10 \mathrm{~cm} \mathrm{H}_{2} \mathrm{O}$ betragen. Der in der Interventionsgruppe erreichte endexspiratorische Druck lag bei $0,1 \mathrm{~cm} \mathrm{H}_{2} \mathrm{O}$, während die nach ARDS Network Trial Tabelle [49] behandelten Patienten einen transpulmonalen Druck von $-2 \mathrm{~cm} \mathrm{H}_{2} \mathrm{O}$ aufwiesen.

Insgesamt lag der PEEP-Wert in der mittels transpulmonalem Druck gesteuerten Gruppe um etwa $4 \mathrm{~cm}$ über dem PEEP-Wert der konventionell behandelten Gruppe.

Die wichtige Botschaft ist hier, nicht generell den PEEP zu erhöhen, da entsprechende Versuche ohne Erfolg blieben [52] sondern die individuelle Pathophysiologie des Patienten zu berücksichtigen und den PEEP genau so einzustellen, dass er endexspiratorisch gerade oberhalb des pleuralen Druckes lieg und so den alveolären Kollaps verhindert. Gleichzeitig wird durch die Strategie ein zu hoher inspiratorischer transpulmonaler Druck vermieden, was Scherkräfte reduziert und so lungenprotektiv wirkt.

\section{Interpretation der Beatmungskurven bei nicht-invasiver Beatmung}

Bei der nichtinvasiven Beatmung können die in $\bullet$ Tab. 2 aufgeführten Phänomene auftreten [54]. Zur Detektion dieser Phänomene ist in der Regel die Durchführung einer polygrafischen Messung erforderlich, da anders als bei der invasiven Beatmung die Atemanstrengungen des Patienten nicht valide an der Druckkurve zu erkennen sind. Autotriggern ( $\bullet$ Abb.6) wird durch einen zu sensibel gewählten Inspirationstrigger ausgelöst. Das fehlerhafte Antriggern kann durch Druckimpulse im Beatmungsschlauch oder in der Beatmungsmaske ausgelöst werden. Hierfür verantwortlich sein können z.B. vibrierendes Material (z.B. eine Gummilippe) der Beatmungsmaske oder auch Wasseransammlungen im Beatmungsschlauch.

Tab. 2 Asynchronität-Phänomene bei der nicht-invasiven Beatmung, modifiziert nach Crescimanno et al. [54].

\begin{tabular}{|l|l|}
\hline Phänomen & Definition \\
\hline Autotriggern & $\begin{array}{l}\text { mehr als drei Atemzüge oberhalb der } \\
\text { patienteneigenen Atemfrequenz }\end{array}$ \\
\hline Doppeltriggerung & $\begin{array}{l}\text { Ein Atemzug des Patienten löst nacheinander } \\
\text { zwei Atemzüge am Beatmungsgerät aus. }\end{array}$ \\
\hline $\begin{array}{l}\text { ineffektive } \\
\text { Triggerung }\end{array}$ & $\begin{array}{l}\text { vom Gerät unbeantwortete Inspirations- } \\
\text { anstrengung des Patienten }\end{array}$ \\
\hline $\begin{array}{l}\text { verlängerte } \\
\text { Insufflation }\end{array}$ & $\begin{array}{l}\text { Die Druckabgabe am Gerät ist zu lang und reicht } \\
\text { bis in die Exspirationsphase des Patienten. }\end{array}$ \\
\hline
\end{tabular}

Doppeltriggern tritt auf, wenn die Inspirationszeit zu kurz gewählt wird. Der Patient atmet am Ende des vom Beatmungsgerät abgegebenen Atemzuges weiter ein und löst einen erneuten Atemzug am Beatmungsgerät aus.

Ineffektive Triggerung bedeutet, dass die inspiratorische Atemanstrengung des Patienten nicht vom Beatmungsgerät beantwortet wird ( $\bullet$ Abb. 7). Die Diskrepanz zwischen der Atemanstrengung (gemessen über den Brustgurt) sowie der gemessenen Druckkurve kann über den Phasenwinkel beider Kurven zuei-

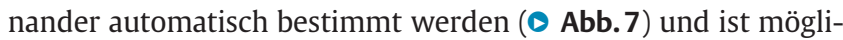
cherweise ein Parameter, mit dem man Phänomene der Asynchronität automatisch quantifizieren kann.

Eine verlängerte Insufflation tritt auf, wenn die Inspirationszeit zu lang oder der Exspirationstrigger zu unsensibel gewählt wurde. Der Patient versucht dann schon auszuatmen, während das Gerät sich noch in der Inspirationsphase befindet. Mögliche Folgen sind eine erschwerte Exspiration und ggf. das Entstehen eines intrinsischen PEEP. 


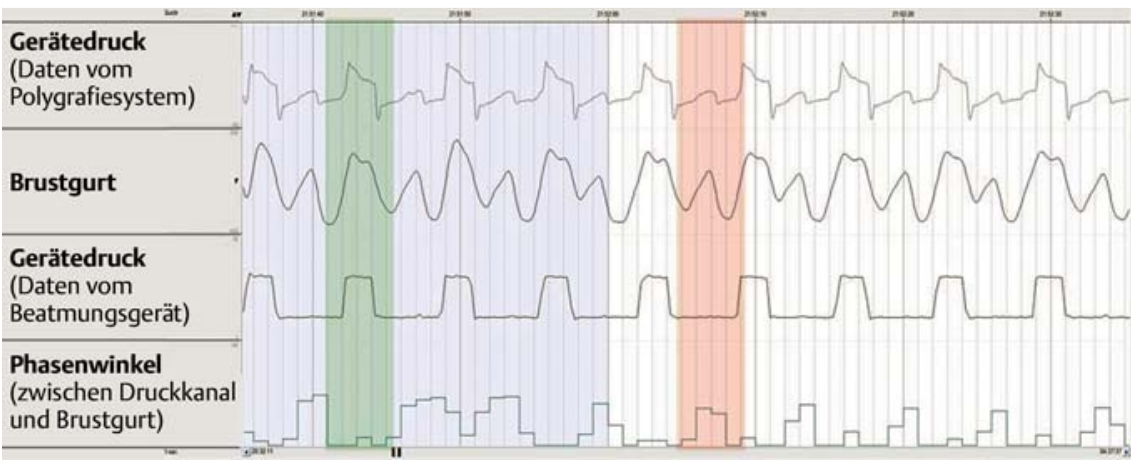

Abb.7 Ineffektive Triggerung: Im grünen Bereich dargestellt ist eine vom Gerät beantwortete Atemanstrengung zu erkennen. Im roten Bereich markiert ist zwar eine Inspirationsbemühung des Patienten erkennbar, eine Antwort des Beatmungsgerätes bleibt jedoch aus. Der Unterschied in den beiden oberen Kurven kann auch über den Phasenwinkel zueinander ausgedrückt werden. Dieser ist ganz unten in der Abbildung dargestellt.

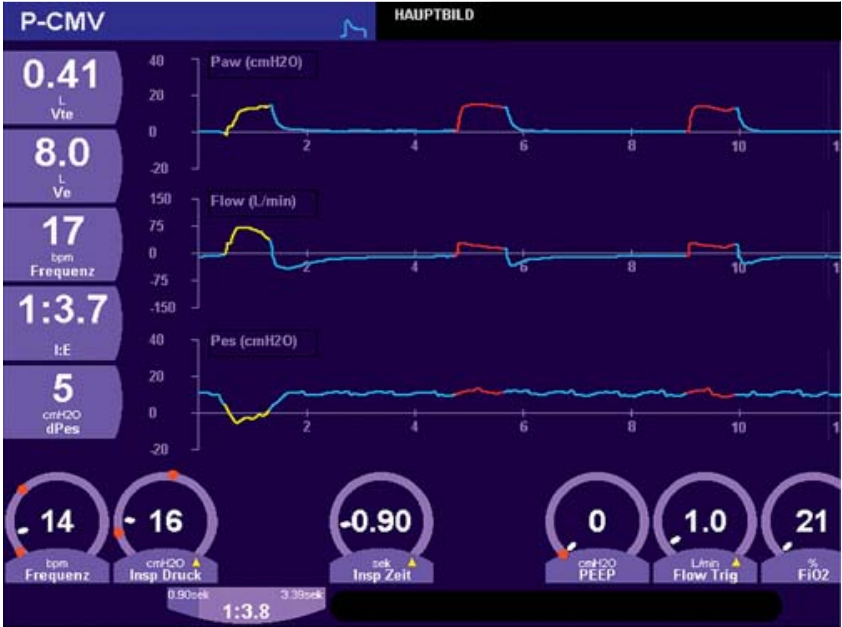

Abb.8 Die vom Patienten getriggerten Atemzüge werden hier in gelber Farbe dargestellt, die mandatorisch vom Gerät abgegebenen in roter Farbe. Die untere Kurve zeigt den Ösophagusdruck über einen liegenden Ösophaguskatheter an (Pes). Bei der aktiven Einatmung durch den Patienten ist ein Druckabfall zu erkennen. Dagegen ist bei den roten, mandatorisch abgegebenen Atemzügen kein Druckabfall zu erkennen, in diesem Falle entsteht bei diesen Atemzügen eine geringere Atemarbeit bei dem Patienten.

\section{Interpretation der Beatmungskurven bei invasiver Beatmung}

Bei der invasiven Beatmung besteht durch den geblockten künstlichen Atemweg eine direkte, in der Regel leckagefreie Verbindung zwischen Beatmungsgerät und Patient, sodass die vom Beatmungsgerät selber aufgezeichneten Druck- und Flusskurven im Vergleich zur nicht-invasiven Beatmung genauer sind und zum Monitoring herangezogen werden können. Moderne Beatmungsgeräte zeigen in der Regel über ihre Grafik an, ob ein Atemzug vom Patienten angetriggert oder mandatorisch vom Beatmungsgerät abgegeben wurde ( $\bullet$ Abb. 8). Die Tatsache, ob ein Atemzug vom Patienten getriggert wurde oder nicht, sagt aber noch nichts über die Atemarbeit des Patienten aus. Auch mandatorisch abgegebene Atemzüge können mit patienteneigener Atemarbeit einhergehen, wenn der Patient zum Beispiel aktiv mitatmet

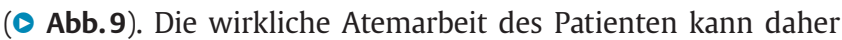
valide nur über die Messung der Atemarbeit mittels Ösophaguskatheter bestimmt werden. Abb.10 zeigt exemplarisch den Unterschied der Atemarbeit bei entlastender kontrollierter und nicht entlastender assistierter Beatmung.

Zur optimalen Einstellung des Inspirationstriggers ist der Druckverlauf des Atemwegsdruckes (Paw) sehr hilfreich. In - Abb.11 kann man einen deutlichen inspiratorischen Abfall

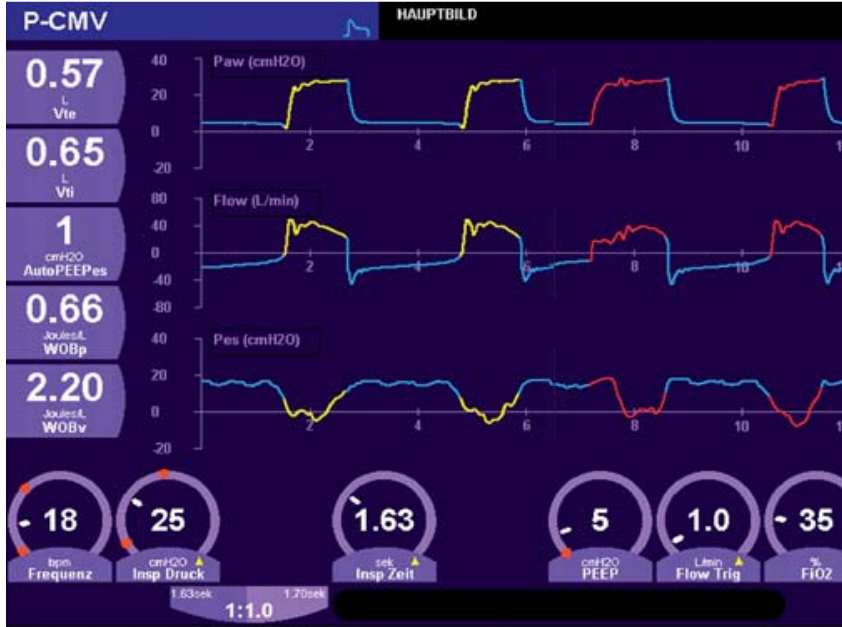

Abb.9 Das Beatmungsgerät klassifiziert assistierte (gelb) und kontrollierte (rot) Atemzüge. Anhand der Ösophagusdruckkurve kann man jedoch hier erkennen, dass im vorliegenden Fall auch bei den kontrollierten Atemzügen ein deutlicher Abfall des intrathorakalen Druckes (Pes) zu erkennen ist, welches die inspiratorischen Anstrengung des Patienten widerspiegelt.

des Atemwegsdruckes bei zu unsensibel eingestelltem Trigger $(\bullet$ Abb.11a) erkennen, während in der Grafik $\bullet$ Abb.11 b bei sensibler eingestelltem Trigger dieses Phänomen nicht mehr zu beobachten ist.

Auch die Steuerung der Inspirationslänge ist von entscheidender Bedeutung. Dies erfolgt entweder über eine inspiratorische Zeitvorgabe bzw. die Einstellung des Exspirationstriggers bei druckkontrollierter Beatmung oder wird bei volumenkontrollierter Beatmung über das vorgewählte Tidalvolumen in Zusammenhang mit der Geschwindigkeit des Druckanstieges bestimmt. Ist die Inspirationszeit zu lang und reicht bis in die Ausatemphase des Patienten, kann dies zum Entstehen eines intrinsischen PEEP führen [55]. Ein intrinsischer PEEP erschwert die Triggerung des nächsten Atemzuges, da der positive intrathorakale Druck durch die nächste Atemanstrengung zunächst auf null reduziert werden muss, bevor durch Unterdruck der nächste Atemzug angetriggert werden kann. Eine inadäquat lange Inspirationszeit erkennt man ggf. an einer endinspiratorischen Druckspitze im Atemwegsdruck, die durch die Exspirationsbemühung des $\mathrm{Pa}$ tienten hervorgerufen wird.

Ist die Inspirationslänge dagegen zu kurz und bricht die Unterstützung des Beatmungsgerätes ab, während der Patient weiter einatmen will, so kann es zu einem mehrfachen Antriggern des 


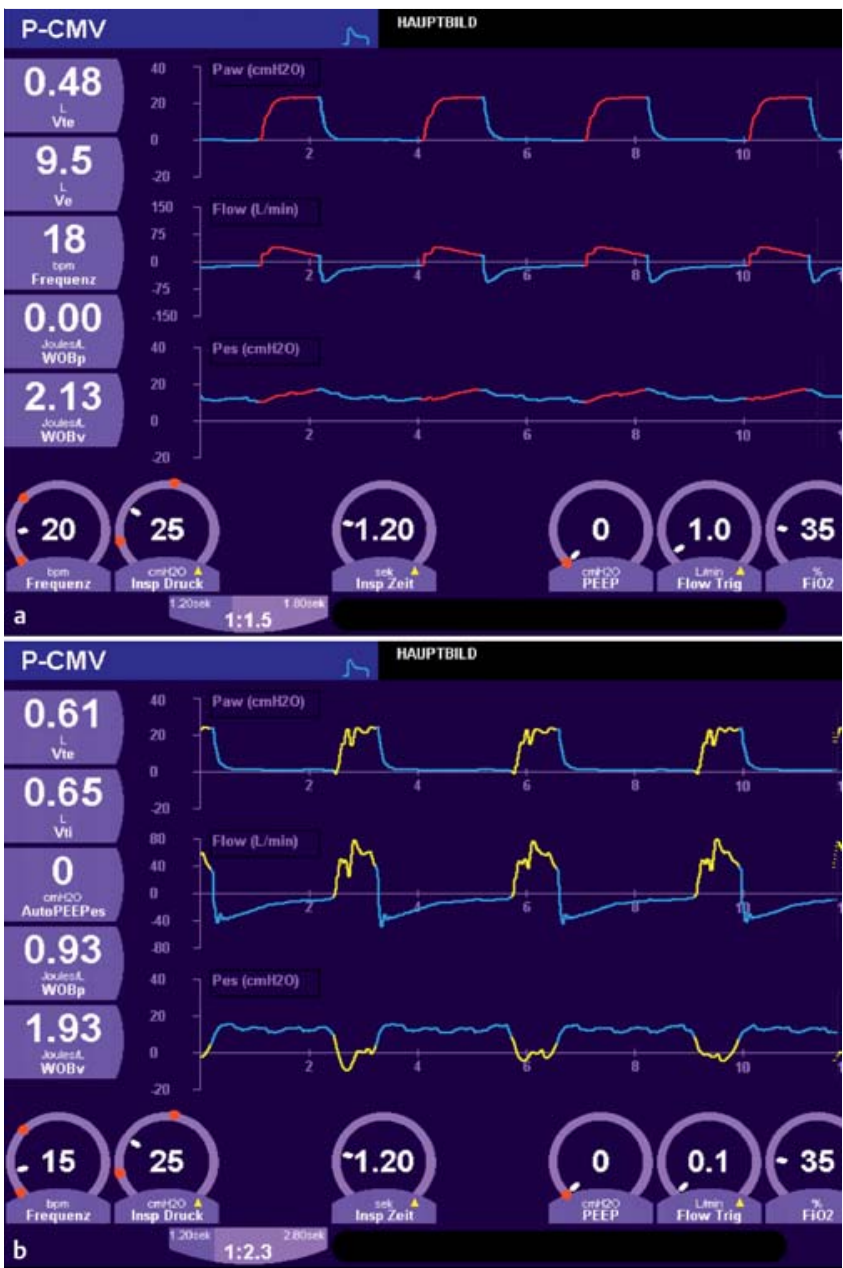

Abb. 10 Teil a zeigt, wie sich bei kontrollierter Beatmung die patienteneigene Atemarbeit (WOBp) auf null reduzieren kann, entsprechend sieht man einen positiven Druckverlauf in der Kurve des Ösophaguskatheters (Pes), das Beatmungsgerät leistet die gesamte Atemarbeit, in diesem Falle 2,13 J/L. Bei assistierter Beatmung (b) sieht man einen inspiratorischen Abfall im Ösophagusdruck, die Atemarbeit des Patienten wurde in diesem Beispiel mit 0,93 J/L bestimmt, während der Ventilator 1,93 J/L leistet (WOBv). Es verbleiben also in etwa 33\% der Atemarbeit beim Patienten.

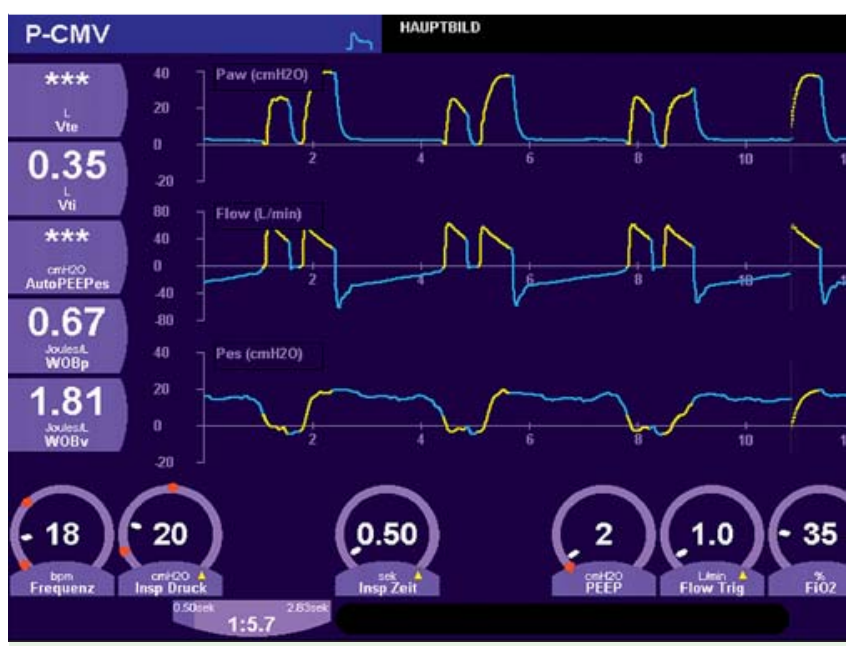

Abb.12 Doppeltriggerung. Hierbei wird wie durch eine zu kurz gewählte Inspirationszeit die Geräteunterstützung zu früh abgebrochen und innerhalb derselben Atemanstrengung des Patienten ein zweiter Atemzug am Gerät ausgelöst.

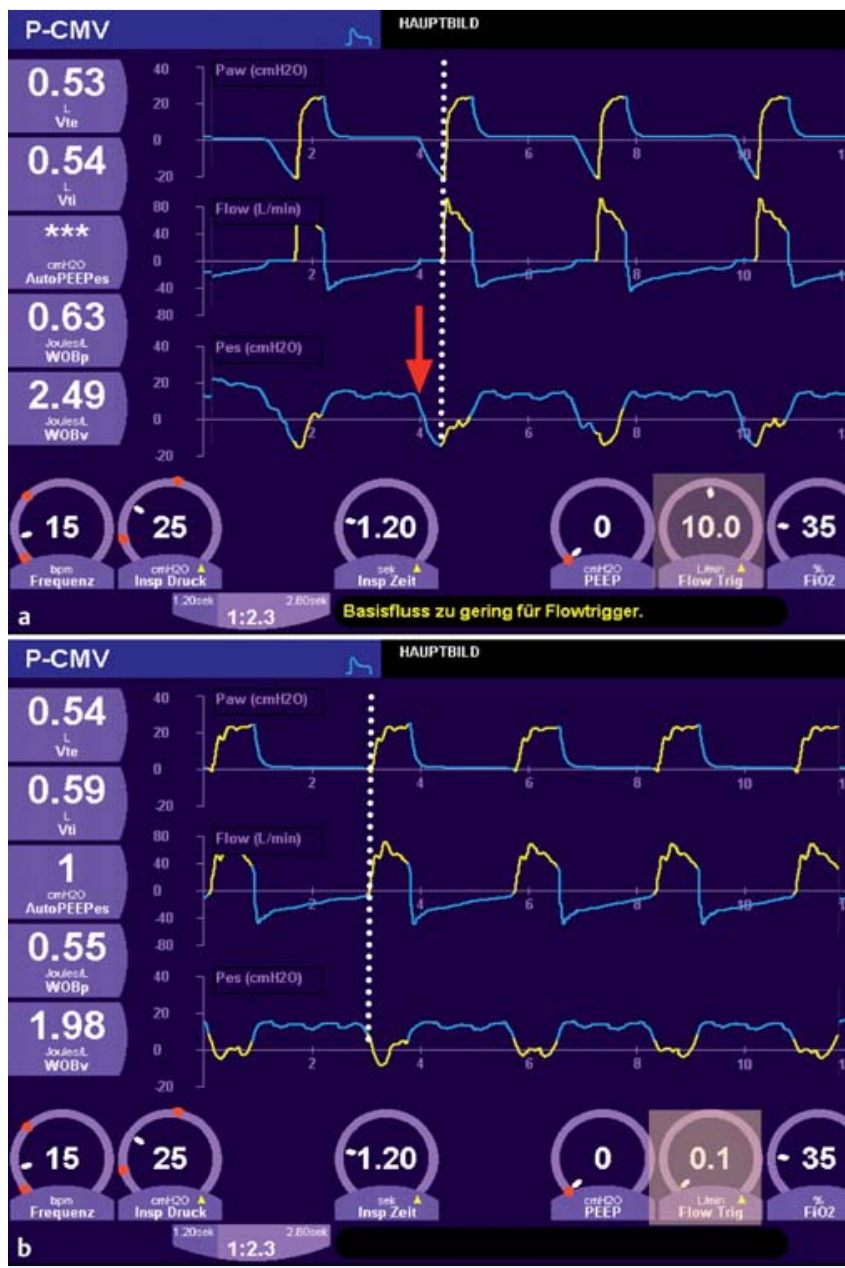

Abb.11 Teil a zeigt, wie bei unsensible eingestelltem Trigger der Atemwegsdruck zu Beginn der Inspiration abfällt. Bei sensibel eingestelltem Trigger (b) findet dieses Phänomen nicht statt. Der genaue Beginn der Atemanstrengung kann anhand der Druckkurve des Ösophaguskatheters beobachtet werden. In Abb. 11 a ist die zeitliche Verzögerung zwischen dem Beginn der Inspirationsanstrengung (roter Pfeil) und dem Beginn der Geräteunterstützung (weiße gestrichelte Linie) zu erkennen. Dagegen ist in Teil b eine gute Synchronität zwischen Atemanstrengung des Patienten und Geräteunterstützung bei sensiblerem Trigger zu erkennen.

Beatmungsgerätes innerhalb derselben Atemanstrengung des Patienten kommen, welches in $\bullet$ Abb. 12 dargestellt ist.

Einige Beatmungsgeräte sind in der Lage Fluss-Volumenkurven anzuzeigen, ähnlich den Kurven der Spirometrie. Obwohl es sich nicht um maximale Manöver handelt wie z. B. bei der Analyse des FEV1 in der Lungenfunktion, kann man doch Rückschlüsse auf das Vorliegen einer obstruktiven Atemwegserkrankung ziehen. - Abb.13 zeigt exemplarisch eine Patientin, bei der eine exspiratorische Flusslimitation erkennbar ist.

Zusammenfassend ist die Interpretation der Beatmungskurven sowohl bei der nicht-invasiven als auch invasiven Beatmung komplex, aber auch entscheidend für eine suffiziente Beatmungstherapie und folglich auch für den Therapieverlauf des Patienten. Die hierbei notwendigen Analysen und Interpretationen sind jedoch von vielen Dingen abhängig. Es müssen neben einer guten Kenntnis der physikalischen Eigenschaften der verwendeten Beatmungstechnik auch die pathophysiologischen Prinzipien der zugrunde liegenden Erkrankung berücksichtigt werden. 


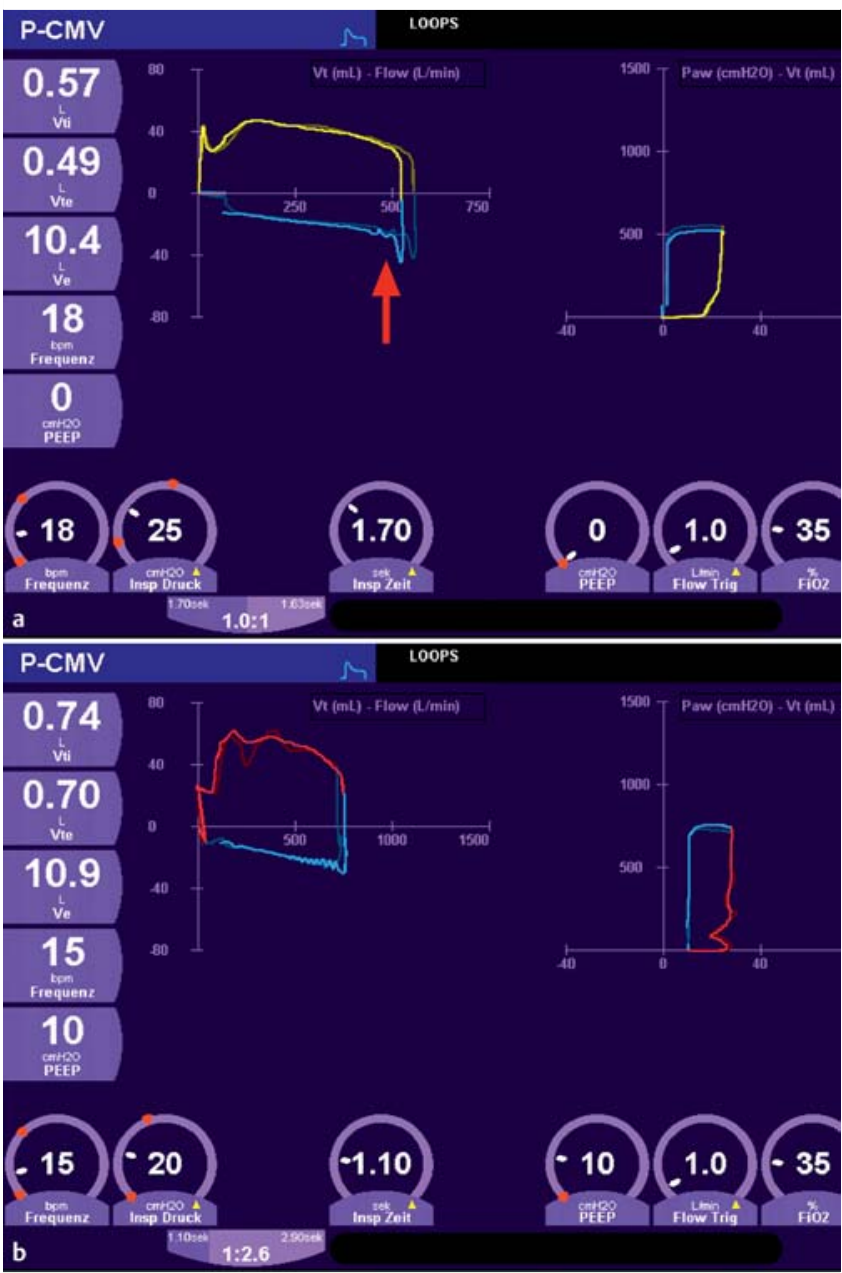

Abb.13 Teil a zeigt eine abrupte Reduktion des Exspirationsflusses (roter Pfeil), der durch den Kollaps der Atemwege zustande kommt, ähnlich wie dem Knick in der Flussvolumenkurve der Spirometrie bei obstruktiven Atemwegserkrankungen. Teil b zeigt beim gleichen Patienten wie über die Hinzunahme eines PEEP (in diesem Falle 10 mbar) dieses Phänomen beseitigt werden kann.

\section{Interessenkonflikt}

$\nabla$

J. H. Storre erhielt Honorare für Vorträge von Breas Medical AB Sweden, Respironics Inc. USA, ResMed Germany Inc.; Heinen und Löwenstein Deutschland, Werner und Müller Medizintechnik, Keller Medical GmbH, Germany, SenTec AG Schweiz, Radiometer GmbH Deutschland, VitalAire GmbH Deutschland. Für Gutachten und Beratertätigkeiten hat J. H. Storre Honorare von Respironics Inc. USA, Breas Medical AB Sweden und Boehringer Ingelheim Pharma GmbH \& Co. KG Deutschland erhalten. J. H. Storre hat zudem Reisekostenunterstützung für nationale and internationale Fachkongresse von Breas Medical GmbH Deutschland, Heinen und Löwenstein Deutschland; Respironics International, Respironics Germany, SenTec AG Switzerland, Vivisol Germany, Weinmann GmbH und Werner und Müller Medizintechnik bekommen. D. Dellweg erhielt Vortragshonorare von Resmed und Heinen und Löwenstein.

\section{Literatur}

1 Kabitz H, Windisch W. Diagnostik der Atemmuskelfunktion: state of the art. Pneumologie 2007; 61: $582-587$

2 Windisch W. Pathophysiologie der Atemmuskelschwäche. Pneumologie 2008; 62 (Suppl. 01): S18-S22

3 Schönhofer B, Kuhlen R, Neumann $P$ et al. Nichtinvasive Beatmung als Therapie der akuten respiratorischen Insuffizienz. Pneumologie 2008; 62: $449-479$

4 Windisch W, Brambring J, Budweiser S et al. Nichtinvasive und invasive Beatmung als Therapie der chronischen respiratorischen Insuffizienz. S2-Leitlinie herausgegeben von der Deutschen Gesellschaft fur Pneumologie und Beatmungsmedizin e.V. Pneumologie 2010; 64: 207 240

5 Severinghaus JW. The invention and development of blood gas analysis apparatus. Anesthesiology 2002; 97: 253-256

6 Storre $\mathrm{JH}$, Steurer $\mathrm{B}$, Kabitz $\mathrm{H}$ et al. Transcutaneous $\mathrm{PCO}_{2}$ monitoring during initiation of noninvasive ventilation. Chest 2007; 132: 1810 1816

7 Zavorsky GS, Cao J, Mayo NE et al. Arterial versus capillary blood gases: a meta-analysis. Respiratory physiology and neurobiology 2007; 155: $268-279$

8 Storre JH, Magnet FS, Dreher $M$ et al. Transcutaneous monitoring as a replacement for arterial $\mathrm{PCO}_{2}$ monitoring during nocturnal non-invasive ventilation. Respiratory medicine 2011; 105: 143 - 150

9 Storre JH. Monitoring der Ventilation bei nächtlicher nichtinvasiver Beatmung. Habilitationsschrift der Medizinischen Universitätsklinik der Albert-Ludwigs-Universität Freiburg im Breisgau: 2011

10 Pitkin $A D$, Roberts $C M$, Wedzicha JA. Arterialised earlobe blood gas analysis: an underused technique. Thorax 1994; 49: 364-366

11 Magnussen $H$, Kirsten A, Kohler $D$ et al. Leitlinien zur Langzeit-Sauerstofftherapie. Deutsche Gesellschaft für Pneumologie und Beatmungsmedizin e.V. Pneumologie 2008; 62: 748 - 756

12 Severinghaus JW, Astrup P, Murray JF. Blood gas analysis and critical care medicine. Am J Respir Crit Care Med 1998; 157: S114-S122

13 Eberhard $P$. The design, use, and results of transcutaneous carbon dioxide analysis: current and future directions. Anesthesia and Analgesia 2007; 105: S48 - S52

14 Paiva $R$, Krivec $U$, Aubertin $G$ et al. Carbon dioxide monitoring during long-term noninvasive respiratory support in children. Intensive Care Med 2009; 35: $1068-1074$

15 Chhajed PN, Heuss LT, Tamm M. Cutaneous carbon dioxide monitoring in adults. Curr Opin Anaesthesiol 2004; 17: 521 - 525

16 Cuvelier A, Grigoriu B, Molano LC et al. Limitations of transcutaneous carbon dioxide measurements for assessing long-term mechanical ventilation. Chest 2005; 127: $1744-1748$

17 Cox M, Kemp R, Anwar S et al. Non-invasive monitoring of CO2 levels in patients using NIV for AECOPD. Thorax 2006; 61: $363-364$

18 Belpomme V, Ricard-Hibon A, Devoir C et al. Correlation of arterial PCO2 and PETCO2 in prehospital controlled ventilation. Am J Emerg Med 2005; 23: $852-859$

19 Yamanaka MK, Sue DY. Comparison of arterial-end-tidal PCO2 difference and dead space/tidal volume ratio in respiratory failure. Chest 1987; 92: $832-835$

20 Sanders $M H$, Kern NB, Costantino JP et al. Accuracy of end-tidal and transcutaneous $\mathrm{PCO}_{2}$ monitoring during sleep. Chest 1994; 106: $472-483$

21 Hinkelbein J, Floss F, Denz C et al. Accuracy and precision of three different methods to determine $\mathrm{Pco} 2$ (Paco2 vs. Petco2 vs. Ptcco2) during interhospital ground transport of critically ill and ventilated adults. J Trauma 2008; 65: 10-18

22 Wilson J, Russo P, Russo J et al. Noninvasive monitoring of carbon dioxide in infants and children with congenital heart disease: end-tidal versus transcutaneous techniques. J Intensive Care Med 2005; 20: 291 295

23 Morgan AP. The pulmonary toxicity of oxygen. Anesthesiology 1969; 30: $587-588$

24 Nichols CW, Lambertsen C. Effects of high oxygen pressures on the eye. N Engl J Med 1969; 281: 25 - 30

25 Smith JL. The pathological effects due to increase of oxygen tension in the air breathed. J Physiol 1899; 24: 19-35

26 Köhler D, Dellweg D. Polyglobulie. Dtsch Med Wochenschr 2010; 135: $2300-2303$

27 Zander $R$. Die kritischen Grenzen der Hamodilution: Theoretische Grundlagen. Beitr Infusionsth 1993; 29: 51 - 69 
28 Schumacker PT, Cain SM. The concept of a critical oxygen delivery. Intensive Care Med 1987; 13: 223-229

29 Mitsuoka M, Kinninger KH, Johnson FW et al. Utility of measurements of oxygen cost of breathing in predicting success or failure in trials of reduced mechanical ventilatory support. Respir Care 2001; 46: 902 -910

30 Bellani $G$, Foti $G$, Spagnolli $E$ et al. Increase of oxygen consumption during a progressive decrease of ventilatory support is lower in patients failing the trial in comparison with those who succeed. Anesthesiology 2010; 113: $378-385$

31 Cain SM, Chapler CK. Effects of norepinephrine and alpha-block on 02 uptake and blood flow in dog hindlimb. J Appl Physiol 1981; 51: $1245-1250$

32 Field S, Sanci S, Grassino A. Respiratory muscle oxygen consumption estimated by the diaphragm pressure-time index. J Appl Physiol 1984; 57: $44-51$

33 Campbell EE, Newsom DavisJ. The respiratory muscles, mechanics and neural control, second ed. London: 1970

34 Yang KL, Tobin MJ. A prospective study of indexes predicting the outcome of trials of weaning from mechanical ventilation. $\mathrm{N}$ Engl J Med 1991; 324: 1445 - 1450

35 Dellweg D, Haidl P, Siemon K et al. Impact of breathing pattern on work of breathing in healthy subjects and patients with COPD. Respir Physiol Neurobiol 2008; 161: 197-200

36 Puddy A, Patrick W, Webster K et al. Respiratory control during volumecycled ventilation in normal humans. J Appl Physiol 1996; 80: 1749 1758

37 Laghi F, SegalJ, Choe WK et al. Effect of imposed inflation time on respiratory frequency and hyperinflation in patients with chronic obstructive pulmonary disease. Am J Respir Crit Care Med 2001; 163 : $1365-1370$

38 Georgopoulos D, Mitrouska I, Webster Ket al. Effects of inspiratory muscle unloading on the response of respiratory motor output to $\mathrm{CO} 2$. Am J Respir Crit Care Med 1997; 155: 2000-2009

39 Sassoon CS. Triggering of the ventilator in patient-ventilator interactions. Respir Care 2011; 56: 39-51

40 Jubran A, Tobin MJ. Pathophysiologic basis of acute respiratory distress in patients who fail a trial of weaning from mechanical ventilation. Am J Respir Crit Care Med 1997; 155: 906-915

41 Sassoon CSH, Caiozzo VJ, Manka A et al. Altered diaphragm contractile properties with controlled mechanical ventilation. J Appl Physiol 2002; 92: 2585-2595

42 Levine $S$, Nguyen T, Taylor $N$ et al. Rapid disuse atrophy of diaphragm fibers in mechanically ventilated humans. N Engl J Med 2008; 358: $1327-1335$
43 Reid WD, Belcastro AN. Chronic resistive loading induces diaphragm injury and ventilatory failure in the hamster. Respir Physiol 1999; 118: $203-218$

44 Jubran A, Van de Graaff WB, Tobin MJ. Variability of patient-ventilator interaction with pressure support ventilation in patients with chronic obstructive pulmonary disease. Am J Respir Crit Care Med 1995; 152 : $129-136$

45 Leung P, Jubran A, Tobin MJ. Comparison of assisted ventilator modes on triggering, patient effort, and dyspnea. Am J Respir Crit Care Med 1997; 155: $1940-1948$

46 Nava S, Bruschi C, Fracchia C et al. Patient-ventilator interaction and inspiratory effort during pressure support ventilation in patients with different pathologies. Eur Respir J 1997; 10: 177-183

47 Van de Graaff WB, Gordey K, Dornseif SE et al. Pressure support. Changes in ventilatory pattern and components of the work of breathing. Chest 1991; 100: 1082-1089

48 Fauroux B, Hart N, Luo YM et al. Measurement of diaphragm loading during pressure support ventilation. Intensive Care Med 2003; 29 : $1960-1966$

49 ARDS Network Trial. Ventilation with lower tidal volumes as compared with traditional tidal volumes for acute lung injury and the acute respiratory distress syndrome. The Acute Respiratory Distress Syndrome Network. N Engl J Med 2000; 342: 1301 - 1308

50 Brower RG, Lanken PN, MacIntyre $N$ et al. Higher versus lower positive end-expiratory pressures in patients with the acute respiratory distress syndrome. N Engl J Med 2004; 351: 327-336

51 Meade MO, Cook DJ, Guyatt GH et al. Ventilation strategy using low tidal volumes, recruitment maneuvers, and high positive end-expiratory pressure for acute lung injury and acute respiratory distress syndrome: a randomized controlled trial. JAMA 2008; 299: 637-645

52 Mercat A, Richard JM, Vielle $B$ et al. Positive end-expiratory pressure setting in adults with acute lung injury and acute respiratory distress syndrome: a randomized controlled trial. JAMA 2008; 299: 646-655

53 Talmor D, Sarge T, Malhotra A et al. Mechanical ventilation guided by esophageal pressure in acute lung injury. N Engl J Med 2008; 359: 2095-2104

54 Crescimanno G, Canino M, Marrone O. Asynchronies and sleep disruption in neuromuscular patients under home mechanical ventilation. Respir Med 2012; 106: 1478-1485

55 Nilsestuen JO, Hargett KD. Using ventilator graphics to identify patientventilator asynchrony. Respir Care 2005; 50: 202 -234 